J. Clin. Chem. Clin. Biochem.

Vol. 15, 1977, pp. 397-399

\title{
Plasminogengehalt in Human-Fibrinogen-Präparationen verschiedener Hersteller ${ }^{1}$ )
}

\author{
Von $H$. Auel und M. Martin
}

Aus der Aggertalklinik, Klinik für Gefäßerkrankungen (Chefarzt: Prof. Dr. W. Schoop) Engelskirchen bei Köln

(Eingegangen am 3. Januar/1. März 1977)

Zusammenfassung: Im Rahmen der fibrinolytischen Therapie interessierte der Plasminogengehalt verschiedener Handelsfibrinogene. Mit Hilfe der semiquantitativen Plasminogenbestimmung war diese Frage beantwortbar. Der ermittelte Plasminogengehält in den Fibrinogenpräperationen wurde der Plasminogenkonzentration in gepooltem Human-Mischplasma gegenübergestellt. Die Relation von Plasminogen zu Fibrinogen betrug im Vergleich zu Human- . plasma für Fibrinogen Immuno 134\%, für Fibrinogen Behringwerke 13,7\% und für Fibrinogen Kabi $2,71 \%$.

\section{Plasminogen content of human fibrinogen preparations from different sources}

Summary: In connection with fibrinolytic therapy, it is of interest to know how much plasminogen is contained in different commercially available fibrinogen preparations. This problem was investigated with the aid of a semiquantitative determination of plasminogen. The determined plasminogen content was compared with plasminogen concentration of pooled human plasma. Using the ratio of plasminogen to fibrinogen, and fixing the plasminogen content of human plasma arbitrarily at $100 \%$, the proportion of plasminogen in fibrinogen from Immuno is $134 \%$, fibrinogen from Behringwerke contains $13.7 \%$, and Kabi-fibrinogen contains $2.71 \%$ plasminogen.

\section{Einführung}

Human-Fibrinogen dient zur Substitution der Fibrinogenfraktion im menschlichen Blut. Ereignet sich z. B. im Rahmen einer fibrinolytischen Therapie bei stark erniedrigter Fibrinogenkōnżentration eine Blutung, so stellt dies eine klare Indikation zur Gabe von Human-Fibrinogen dar. Von großer klinischer Bedeutung ist hierbei die Frage, wieviel Plasminogen mit dem Fibrinogen zugeführt wird. Bei nur kurzzeitiger Unterbrechung der Streptokinaseinfusion treffen die noch zirkulierenden Streptokinasequantitäten auf die frisch infundierte, plasminogenhaltige Fibrinogenpräparation. Unter dieser Bedingung kann es zur Umwandlung in Plasinin mit nachfolgender Fibrinogenolyse und Auftreten unerwünschter gerinnungshemmender Fibrinógenspaltprodukte kommen.

Es ergab sich somit die klinisch wichtige Frage, wieviel Plasminogen einzelnen kommerziell angebotenen HumanFibrinogen-Präparationen anhaftet. Bei hohem Plasmino-

\footnotetext{
1) Mit Unterstïtzung des Vereins zự Bekämpfung der Gefäßkrankheiten e. V., D-5250 Engelskirchen, Aggertalklinik.
}

gengehalt wäre nach Fibrinogen-Infusion mit einer erheblichen unerwünschten Plasminämie zu rechnen.

In der folgenden Studie wird der Plasminogengehalt in Human-Fibrinogen-Präparaten verschiedener Hersteller ermittelt und auf den Fibrinogengehalt der einzelnen Proben bezogen.

\section{Material und Methode}

Plașminọen-Bestim mung

Reagenzien und technische Ausrüstung

1. Rinderfibrinogen, Fläschchen zu $60 \mathrm{mg}$, Behringwerke AG, Marbür/Lahn.

2. Test-Streptokinase, Fläschchen zu $5000 \mathrm{E}$, Behring werke AG, Marburg/Lahn.

3. Thrombin, Ampulle zu $16 \mathrm{E}$, Antithrombin-Reagenz, Hoffmann-La Roche AG, Grenzach/Baden.

4. Michaelispuffer $\mathrm{pH}^{7} 7,4$ und $\mathrm{pH} 7,8$ (Ionenstärke 0,1).

5. Citrat-Mischplasma ( 9 Teile Blut, 1 Teil Natriumcitrat 3,8\%, zentrifugiert bei $2500 \mathrm{U} / \mathrm{min}$ für $10 \mathrm{~min}$ ) von 30 Probanden (Patienten mit statischen Beschwerden). 
6. Human-Fibrinogen der Deutschen Kabi GmbH, München, Behringwerke AG, Marburg/Lahn, Immuno GmbH, Heidelberg.

7. Thrombe lastographie-Ausrüstung nach Hartert (1), Fa. Hellige, Freiburg/Breisgau.

Methodische Einzelheiten zur Plasminogen-Bestimmung nach Martin $(2,3)$

Der Inhalt eines Fläschchens Rinderfibrinogen wurde in ein Reagenzglas überführt, mit 6,0 ml Michaelis-Puffer pH 7,8 überschichtet und zur Lösung für 30 Minuten im Wasserbad bei $37^{\circ} \mathrm{C}$ belassen. Das so gelöste Rinderfibrinogen wurde vor Gebrauch filtriert. Ferner kamen 2 Fläschchen Test-Streptokinase in je $0,625 \mathrm{ml}$ Michaelis-Puffer $\mathrm{pH} 7,4$ und 1 Ampulle Thrombin in 1,0 ml Michaelis-Puffer pH 7,4 zur Auflösung.

Der Inhalt einer Infusionsflasche mit $1 \mathrm{~g}$ Human-Fibrinogen wurde in $50 \mathrm{ml}$ Lösungsmittel (dest. Wasser bzw. physiol. $\mathrm{NaCl}$-Lösung) aufgenommen; es resultierte eine Konzentration von $1000 \mathrm{mg}$ Fibrinogen pro $50 \mathrm{ml}$ Lösungsmittel $(=20 \mathrm{~g} / \mathrm{l})$. Durch eine weitere 1:10 Verdünnung ergab sich eine „Firmendeklarierte Fibrinogenkonzentration" von $2 \mathrm{~g} / \mathrm{l}$.

Citrat-Mischplasma von 30 Probanden wurde 1:40 und 1:4000 mit Michaelis-Puffer pH 7,4 verdünnt. Die Verdünnung 1:40 entsprach einer Plasminogenkonzentration von $100 \%$ der Norm, die Verdünnung 1:4000 einer Plasminogenkonzentration von $1 \%$ der Norm.

$0,10 \mathrm{ml}$ Rinderfibrinogenlösung

$0,05 \mathrm{ml}$ Plasmaverdünnung

$0,05 \mathrm{ml}$ Streptokinaselösung

$0,05 \mathrm{ml}$ Thrombinlösung

wurden je zweimal (= Doppelbestimmung) in einer Thrombelastographie-Küvette zur Gerinnung gebracht und die Lysezeit dieses Testgerinnsels registriert. Da bis auf die Plasmaverdünnung alle Komponenten des Testgerinnsels standardisiert waren, hing die Lysezeit allein von der vorhandenen Plasminogenkonzentration im Plasma ab (hohe Plasminogenkonzentrationen - kurze Lysezeiten; niedrige Plasminogenkonzentrationen - lange Lysezeiten). Die erhaltenen spindelförmigen Thrombelastographie-Aufzeichnungen wurden ausgemessen, wobei die Strecke zwischen Auseinanderweichen der Branchen um $1 \mathrm{~mm}$ bis zur Wiederannäherung auf $1 \mathrm{~mm}$ bestimmt wurde. Die ermittelten Werte in $\mathrm{mm}$, dividiert durch 2 ergaben die Lysezeiten in Minuten. Das Eintragen der erhaltenen Werte erfolgte in doppeltlogarithmiertem Papier, wobei auf der Abszisse der Verdünnungsgrad in Prozent und auf der Ordinate die Lysezeit in Minuten erschienen. Die Verbindung der Werte miteinander ergab die Standardgerade (Abb. 1).

Die einzelnen, wie oben beschrieben hergestellten Fibrinogenlösungen $(2 \mathrm{~g} / \mathrm{l})$ mit unbekanntem Plasminogengehalt wurden mit Michaelis-Puffer $\mathrm{pH}$ 7,4 im Verhältnis 1:40 verdünnt und in

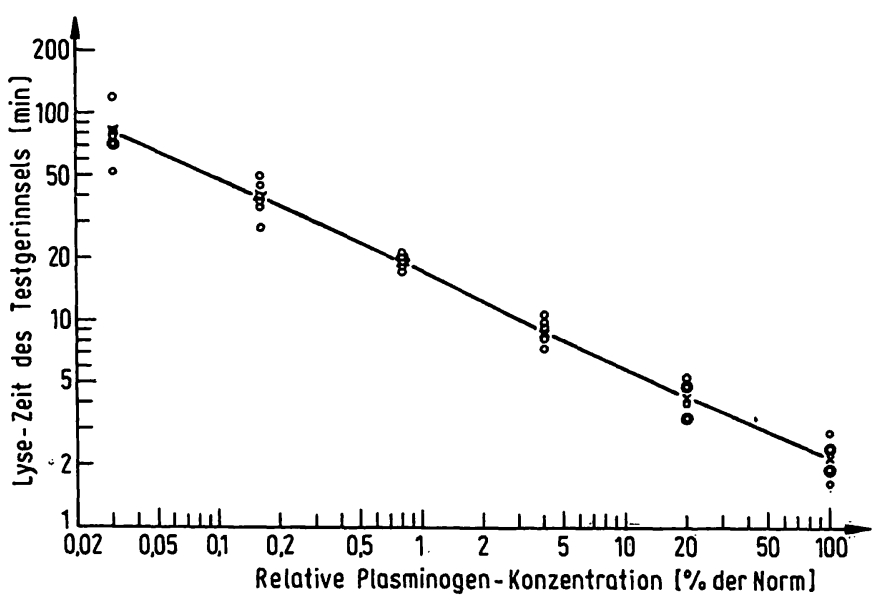

Abb. 1. Standardgerade zur Plasminogen-Bestimmung im doppeltlogarithmischen System. Auf der Abszisse erfolgte die Eintragung der relativen Plasminogenkonzentrationen das Standardgerinnsel eingebracht (Doppelbestimmungen aus einer Verdünnung). Die erhaltenen, auf dem Thrombelastogramm abzulesenden Lysezeiten in Minuten wurden an Hand der zuvor erstellten Standardkurve in Plasminogen umgerechnet.

Fibrinogenbestimmung

Um den gravimetrischen Fibrinogengehalt der einzelnen Fibrinogenpräparate zu erhalten, wurde die Methode nach Gram (4) gewählt. Hierzu wurde der Flascheninhalt $(1 \mathrm{~g}$ Fibrinogen lt. Firmenangabe) in $50 \mathrm{ml}$ Medium gelöst (d. h. eine firmendeklarierte Fibrinogenkonzentration von $20 \mathrm{~g} / \mathrm{l}$ hergestellt), dann nochmals 1:10 weiter verdünnt und in dieser Lösung de Fibrinogengehalt mit der gravimetrischen Methode überprüft. $2.0 \mathrm{ml}$ Fibrinogenlösung $(2 \mathrm{~g} / \mathrm{l})$ wurden mit $0,1 \mathrm{ml}$ Thrombin (50 NIH-E/ml) zum Gerinnen gebracht, $2 \mathrm{~h}$ im Wasserbad bei $37^{\circ} \mathrm{C}$ belassen, anschließend mit physiol. $\mathrm{NaCl}$-Lösung und dest. Wasser ausgewaschen und in absol. Alkohol und Äther entwässert. Zur vollkommenen Trocknung wurde das Fibrin für $12 \mathrm{~h}$ im Brutschrank bei $37^{\circ} \mathrm{C}$ aufbewahrt und dann auf einer Analysenwaage ausgewogen.

\section{Ergebnisse}

Fibrinogengehalt in $4 \mathrm{Handelsfibrinogenlösungen}$ Die gravimetrisch bestimmte Fibrinogenkonżentration wurde in Lösungen bestimmt, die entsprechend den Angaben der Hersteller eine Konzentration von $2 \mathrm{~g} / 1$ enthielten. Die ermittelten Werte sind in der Tabelle 1, Spalte II, $z u$ entnehmen.

Fibrinogenkonzentration von gepooltem Humanplasma

In einem Mischplasma von 30 Probanden wurde mit Hilfe der gravimetrischen Methode eine Fibrinogenkonzentration von $3,55 \mathrm{~g} / 1$ ermittelt.

Plasminogengehalt einer firmendeklarierten Fibrinogenlösung $(2 \mathrm{~g} / 1)$

\section{Plasminogengehalt von Human-Fibrinogen Kabi}

Human-Fibrinogenlösungen vier verschiedener Chargen wurden ausgetestet. Die ermittelten Plasminogenwerte bei deiklarierten Fibrinogenkonzentrationen von $2 \mathrm{~g} / 1$ sind in der Tabelle 1, Spalte I, zu entnehmen.

\section{Plasminogengehalt von Human-Fibrinogen Behring}

Human-Fibrinogenlösungen fünf verschiedener Chargen wurden ausgetestet. Die ermittelten Plasminogenwerte bei deklarierten Fibrinogenkonzentrationen von $2 \mathrm{~g} / 1$ sind der Tabelle 1, Spalte I, zu entnehmen.

\section{Plasminogengehalt von Human-Fibrinogen Immuno}

Human-Fibrinogenlösungen vier verschiedener Chargen wurden ausgetestet. Die ermittelten Plasminogenwerte bei deklarierten Fibrinogenkonzentrationen von $2 \mathrm{~g} / \mathrm{l}$ sind in der Tabelle 1, Spalte I, zu entnehmen.

Plasminogengehalt in kommerziellen Humanfibrinogen-Lösungen, bezogen auf eine normale Plasma-Fibrinogenkonzentration von $3,55 \mathrm{~g} / \mathrm{l}$.

Um die Plasminogenkonzentration in den Fibrinogenlösungen mit jener im Humanplasma korrelieren zu 
Tab. 1. Plasminogengehalt verschiedener Clargen von Fibrinogenpräparaten dreier Hersteller, bezogen auf Fibrinogen. Der Plasminogengehalt eines Mischplasmas $(n=30)$ mit 3,55 g/1 Fibrinogen wurde gleich $100 \%$ gesetzt.

\begin{tabular}{|c|c|c|c|}
\hline Chargen & $\begin{array}{l}\text { Plasminogen- } \\
\text { gehalt in } \\
\text { in deklarierten } \\
\text { Fibrinogen- } \\
\text { lösungen } \\
\text { von } 2 \mathrm{~g} / \mathrm{l}\end{array}$ & $\begin{array}{l}\text { Gravimetrisch } \\
\text { ermittelte } \\
\text { Fibrinogen- } \\
\text { konzentration } \\
\text { in deklarierten } \\
\text { Fibrinogen- } \\
\text { lösungen } \\
\text { von } 2 \mathrm{~g} / 1\end{array}$ & $\begin{array}{l}\text { Umgerechneter } \\
\text { Plasminogen- } \\
\text { gehalt für eine } \\
\text { Fibrinogen- } \\
\text { konzentration } \\
\text { von } 3,55 \mathrm{~g} / 1\end{array}$ \\
\hline \multicolumn{4}{|l|}{ Kabi } \\
\hline $\begin{array}{l}706533492 \\
704533455 \\
705533482 \\
704532677\end{array}$ & $\begin{array}{l}0,98 \% \\
1,80 \% \\
1,45 \% \\
2,00 \%\end{array}$ & $\begin{array}{l}2,00 \mathrm{~g} / 1 \\
1,95 \mathrm{~g} / 1 \\
2,10 \mathrm{~g} / 1 \\
2,10 \mathrm{~g} / 1\end{array}$ & $\begin{array}{l}1,74 \% \\
3,28 \% \\
2,45 \% \\
3,38 \%\end{array}$ \\
\hline \multicolumn{4}{|l|}{ Behring } \\
\hline $\begin{array}{r}1152 \\
1056 \\
1156 \\
1151 \\
10510\end{array}$ & $\begin{array}{r}10,50 \% \\
10,50 \% \\
8,00 \% \\
8,50 \% \\
8,50 \%\end{array}$ & $\begin{array}{l}2,40 \mathrm{~g} / 1 \\
2,60 \mathrm{~g} / 1 \\
2,30 \mathrm{~g} / 1 \\
2,25 \mathrm{~g} / 1 \\
2,30 \mathrm{~g} / 1\end{array}$ & $\begin{array}{l}15,5 \% \\
14,3 \% \\
12,3 \% \\
13,4 \% \\
13,1 \%\end{array}$ \\
\hline \multicolumn{4}{|l|}{ Immuno } \\
\hline $\begin{array}{l}040275 \\
043474 \\
044574 \\
043775\end{array}$ & $\begin{aligned} 86,0 & \% \\
70,0 & \% \\
100,0 & \% \\
100,0 & \%\end{aligned}$ & $\begin{array}{l}2,38 \mathrm{~g} / 1 \\
2,44 \mathrm{~g} / 1 \\
2,50 \mathrm{~g} / 1 \\
2,16 \mathrm{~g} / 1\end{array}$ & $\begin{array}{ll}128,2 & \% \\
101,8 & \% \\
142,0 & \% \\
164,3 & \%\end{array}$ \\
\hline & & & $M=134,0 \%$ \\
\hline
\end{tabular}

können, bezogen wir alle Plasminogenkonzentrationen auf den gravimetrisch ermittelten Fibrinogengehalt eines gepoolten Humanplasmas $(3,55 \mathrm{~g} / \mathrm{l})$ und setzten dessen Plasminogengehalt gleich $100 \%$.

Beispiel: Im Versuch wurde ermittelt, daß einer Fibrinogenlösung von $2 \mathrm{~g} / 10,98 \%$ Plasminogen anhaftete. Bezogen auf einen normalen Plasma-Fibrinogengehalt von 3,55 g/l errechnet sich folgender Plasminogenwert:

$$
\begin{aligned}
\frac{0,98 \%}{2,00 \mathrm{~g} / 1} & =\frac{\mathrm{x} \%}{3,55 \mathrm{~g} / 1} \\
\mathrm{x} & =1,74
\end{aligned}
$$

Eine Fibrinogenlösung von $3,55 \mathrm{~g} / \mathrm{l}$ enthielt somit $1,74 \%$ Plasminogen (siehe Tabelle).

\section{Besprechung und Schlußfolgerung}

Im Rahmen der fibrinolytischen Therapie war von Interesse, wieviel Plasminogen verschiedenen Handels-Fibrinogenen anhaftete.

Mit Hilfe der semiquantitativen Plasminogenbestimmung wurde versucht, diese Frage zu beantworten. Die hier verwendete Methode beruhte auf der Lysezeitbestimmung eines Standardgerinnsels, welches definierte Mengen von Rinderfibrin, Rinderplasminogen, Streptokinase, Humanplasminogen und Thrombin enthielt. Die gemessene Zeit von der Gerinnselbildung bis zur Auflösung war dabei ein reziprokes Maß für den Plasminogengehalt der zugeführten Lösung.

Der Plasminogengehalt von Handelsfibrinogenen wurde mit dem im menschlichen Plasma verglichen. Es zeigte sich, daß Fibrinogen Immuno mit $134 \%$ den höchsten Plasminogenanteil enthielt. Im Vergleich zu Mischplasma mit einer Plasminogenkonzentration von 100\% bedeutet dies, daß Plasminogen und Fibrinogen ohne Trennung voneinander im Herstellungsprozeß gefällt wurden. Im Gegensatz hierzu war Fibrinogen Kabi annähernd plasminogenfrei. Es enthielt lediglich einen Plasminogenanteil von $2,71 \%$.

Fibrinogen Behring stand mit einer Plasminogenkonzentration von $13,7 \%$ etwa in der Mitte zwischen diesen beiden Extremen.

Aus den Untersuchungen ergibt sich, daß zur Blutstillung während einer fibrinolytischen Therapie mit Streptokinase Fibrinogen Kabi am besten geeignet ist. Hier dürfte am wenigsten mit einer erneuten Plasminbildung durch den Kontakt von Plasminogen und Streptokinase gerechnet werden.

\section{Literatur}

1. Hartert, H. (1951), Z. Gesamte Exp. Med. 117, 189-203.

2. Martin, M. (1969), Thromb. Diath. Haemorrh. 22, 121-137.
3. Martin, M. (1976), Thrombos. Haemostas. 36, 551-565.

4. Gram, H. C. (1921), J. Biol. Chem. 49, 279-295. 
